\title{
Draugen Field Development: The Role of Gravity Drainage and Horizontal Wells
}

\author{
Reinholdtsen B.
}

Norske Shell a. s., Norway

\begin{abstract}
Copyright 1985, Steering Committee of the European IOR - Symposium.
This paper was presented at the 8th. European IOR - Symposium in Vienna, Austria, May 15 - 17,1995

This paper was selected for presentation by the Steering Committee, following review of information contained in an abstract

submitted by the author(s). The paper, as presented has not been reviewed by the Steering Committee.
\end{abstract}

\section{Abstract}

The Draugen field consists of an elongated low relief anticline containing mainly multi-Darcy sands. Oil initially in place was 182 million $\mathrm{Sm}^{3}$.

The 1987 Plan for Development and Operation (PDO) assumed a central cluster of 6 deviated production wells and 3 subsea water injectors at each end of the field. In addition two subsea wells would provide early production. Unsteady state relative permeabilities indicated a residual oil saturation of $35 \%$. In the full field simulation model this was increased to $40 \%$ to account for small scale hetrogeneities. The model yielded a recovery of some 67 million $\mathrm{Sm}^{3}$.

Modern steady state and centrifuge techniques have since demonstrated a long tail of very low oil relative permeabilities before reaching a residual oil saturation of some $15 \%$. Fine grid simulations translated this into a gravity segregation process behind the displacement front.

Other fine grid models revealed that horizontal wells would yield a slower build-up of water cut than vertical wells. The increased productivity of the horizontal wells also reduced the required number of platform producers from 6 to 5 .

Pseudo relative permeabilities were developed to represent fine grid model performances in a new sophisticated full field mode. This model predicted an ultimate recovery of 94 million $\mathrm{Sm}^{3}$ ( $40 \%$ increase). The additional recovery is attributed to a reduction in the effective residual saturation and to reduced and delayed build-up of water production.

In October 1993 Draugen started production from one horizontal subsea well, which has since demonstrated a better than expected coning performance. Production from platform wells was initiated in June 1994 with initial well rates up to $8000 \mathrm{Sm}^{3} / \mathrm{d}$, confirming the expected high productivities of horizontal wells.

\section{Introduction}

The Draugen field is located in block $6407 / 9$ in the southern part of the Haltenbanken area, offshore mid-Norway as seen in Fig. 1. The initial field development was finalised in December 1994 with the the completion of the third platform producer. The outline of the field and the main field installations are shown in Fig. 2.

The field was discovered in June 1984 by the first exploration well in the block. Five appraisal wells were drilled on the field during the next two years. The Plan for Development and Operations (PDO) was approved by the Storting (Parliament) in December 1988. The PDO assumed an annual average plateau rate of $14,300 \mathrm{Sm}^{3} / \mathrm{d}$. On this basis the so-called sliding scale was invoked, increasing the State's (including Statoil) interest in Draugen from 50\% to $65 \%$. This lead to the present participation, which is: 


$\begin{array}{ll}\text { Norske Shell (operator) } & 21.0 \% \\ \text { The Norwegian State } & 45.4 \% \\ \text { Statoil } & 19.6 \% \\ \text { BP Norge } & 14.0 \%\end{array}$

The development as presented in the PDO was based on flank injection water drive. In spite of excellent reservoir properties the recovery from the field was estimated at only 67 million $\mathrm{Sm}^{3}$ of the 182 million $\mathrm{Sm}^{3}$ STOIIP. This modest recovery of $37 \%$ was strongly affected by the surprisingly high residual oil saturation of some $35 \%$ indicated by conventional unsteady state water flood experiments. A research project was therefore undertaken at RESLAB in Trondheim to investigate the validity of the unsteady state measurements. Before development an in-house study was also carried out to evaluate the potential benefits of drilling the production wells as horizontal wells instead of deviated wells as assumed in the PDO.

This paper describes the main results of the RESLAB research project and of the horizontal well study. The methods used to implement these results in the full field simulation model and the corresponding field scale effects are also covered.

\section{Field Description}

\subsection{Geology}

Draugen is a geologically uncomplicated field. It consists of a low relief anticline shaped structure, some $21 \mathrm{~km}$ long and $6 \mathrm{~km}$ wide with a maximum vertical closure of some $\mathbf{4 0}$ metres. A structue map is shown in Fig. 3, which also indicates the area included in the full field simulation model. The structure is formed by a simple shallow marine sand bar, underlain and overlain by shale. This sand bar is denoted the Rogn formation and at the centre it appears as a high quality coarsening upwards sequence. It develops from fine silts at the base to highly permeable medium to coarse grained sands, in which the bulk of the hydrocarbons are found.

The Lower Spekk shale underlies the Rogn formation and forms the separation between Rogn and the regionally extensive and highly permeable Garn formation which is water bearing. A type log from the crestal discovery well is shown in Fig. 4. In the western part of the field both Rogn and Lower Spekk pinch out and the Garn formation rises above the OWC. This creates the so-called Garn West substructure, containing some $10 \%$ of the oil in place in Draugen. The degree of communication between Garn West and the main field is uncertain. The communication between Rogn and the underlying Garn aquifer was considered a main uncertainty in the development planning. However, pressure performance during production has now demonstrated some degree of such communication to exist, probably through local holes in the Lower Spekk shale.

\subsection{Rock Properties}

The Draugen rock properties are generally excellent. Porosities exceed $27 \%$ in the entire oil bearing Rogn formation and are in the order of $30 \%$ in the central part of the field. The net to gross ratio exceeds $97 \%$ in the entire Rogn I unit and averages some $50 \%$ in the Rogn II unit. Permeability data are available from cores and production tests in all six exploration/appraisal wells. Typical air permeabilities, which generally are in the Darcy range in the Rogn I unit, are plotted in Fig. 4 for the discovery well.

The tests have proved difficult to interpret, but appear to give higher permeabilities than arithmetically averaged core data corrected for overburden. For simulation modelling purposes an average of core and test derived permeabilities has been applied. To honour the coarsening upward character of Rogn I it was subdivided into 7.3 metre thick sublayers with top Rogn II as the base. This implies that the areal extent of the layers becomes smaller at increasing distances above Rogn II, so that uppermost layer only covers the ridge of the structure. For vertical permeabilities geometric averaging has been assumed. Harmonic averaging, which assumes a stratified system is perceived as too pessimistic for Draugen type sands. On this basis $k_{\mathrm{v}} / \mathrm{k}_{\mathrm{h}}$ ratios of 0.35 and 0.05 have been applied for Rogn I and Rogn II, respectively.

\subsection{Initial Conditions}

The Draugen reservoir is filled to spill point and contains 182 million $\mathrm{Sm}^{3}$ of highly undersaturated oil at hydrostatic pressure of 165 bara. The reservoir oil quality varies across the field with GORs ranging from $67 \mathrm{Sm}^{3} / \mathrm{Sm}^{3}$ in the north to $51 \mathrm{Sm}^{3} / \mathrm{Sm}^{3}$ in the south. The corresponding bubble point pressures are 65 and 34 bara. This variation in fluid properties is explained by migration of different quality hydrocarbons over time from the north-west and various degrees of bio-degradation. The gradual variation is not believed to be associated with reservoir discontinuities. In fact recent production experience has proven pressure communication across the entire field. 
Significant capillary transition zones in the Rogn formation have been observed in the Draugen exploration and appraisal wells, as seen in Fig. 5. Well 6407/9-4, which found oil in the Garn formation, hardly has a transition zone. Drainage capillary pressures based on these observations were used for initialising simulation models.

\section{Relative Permeabilities}

At the time of the PDO relative permeabilities based on conventional unsteady state techniques using viscous oils, were available. In the period 1987 to 1992 RESLAB in Trondheim carried out a research project for Norske Shell on relative permeabilities for Draugen. During this project a number of new experiments were carried out, using various techniques, including steady state and centrifuge. The results from the latter types of experiments have had a profound impact on the evaluation of Draugen reservoir performance and recovery.

In the unsteady state experiments the flooding had to be terminated. when the viscous oil residual saturation was still well above $20 \%$. Potential film flow, which could have further reduced saturation. would not occur at detectable rates. In the steady state and centrifuge experiments a light refined oil, with viscosity of some $2 \mathrm{cp}$ was used. This yielded a viscosity ratio $\mu_{w} / \mu_{0}$, similar to that in the reservoir and resulted in residual saturations in the order of $15 \%$. However, the oil and water viscosities are still more than twice the values at reservoir conditions, possibly resulting in still too high residuals.

A problem in all Draugen relative permeability experiments has been the establishment of correct initial water saturations. Values around $20 \%$ have been achieved, whereas the log derived connate water saturation amounts to less than $10 \%$. At the time of the PDO the amount of movable oil, as indicated by the unsteady state experiments, was used directly. However, the moveable saturation range was shifted to be consistent with the log derived initial water saturation of $10 \%$. This yielded a residual oil saturation of $35 \%$. However, Land ${ }^{1}$ and Honarpour ${ }^{2}$ have demonstrated that the residual oil saturation will only be increased by a small fraction of the increase in oil saturation at the start of the experiment. Thus the amount of movable oil can be significantly increased when the experimental results are corrected for the high initial water saturation. The relationship proposed by Land is presently being applied for this purpose.
The measured steady state and centrifuge data are plotted on a logarithmic relative permeability scale in Fig. 6 . The centrifuge data essentially appear as an extension of the steady state data. Under the high centrifugal forces oil could be extracted out of the core to yield low residual oil saturations ranging from 9 to $17 \%$. Oil production at extremely low relative permeabilities is required to reach these low residuals. Fig. 6 also shows that Corey exponents $n_{0}=3.6$ and $n_{w}=2.0$ give a good match of experimental data. These are based on Corey equations of the form

$$
\begin{aligned}
& \mathrm{k}_{\mathrm{ro}}=\mathrm{k}_{\mathrm{ro}}\left(\mathrm{S}_{\mathrm{wc}}\right) \cdot\left[1-\frac{\mathrm{S}_{\mathrm{w}}-\mathrm{S}_{\mathrm{wc}}}{1-\mathrm{S}_{\mathrm{or}}-\mathrm{S}_{\mathrm{wc}}}\right]^{\mathrm{n}_{\mathrm{o}}} \\
& \mathrm{k}_{\mathrm{rw}}=\mathrm{k}_{\mathrm{rw}}\left(\mathrm{S}_{\mathrm{or}}\right) \cdot\left[\frac{\mathrm{S}_{\mathrm{w}}-\mathrm{S}_{\mathrm{wc}}}{1-\mathrm{S}_{\mathrm{or}}-\mathrm{S}_{\mathrm{wc}}}\right]^{\mathrm{n}_{\mathrm{w}}}
\end{aligned}
$$

A Corey model with the above exponents and a deemed practical residual oil saturation of $20 \%$ is used for fine grid simulations to ensure smooth data. However, two fundamental corrections were made before use in the simulator. Firstly the Land formula was applied to adjust the connate water saturation to the log based value of $10 \%$. Secondly, to account for small scale reservoir hetrogeneities, beyond the resolution of simulator grid blocks, the residual oil saturation was increased from 20 to $25 \%$. Following these corrections the new relative permeability model is plotted on a linear. scale in Fig. 7, which also shows the comparison with the old PDO model.

In the centrifuge experiments high negative capillary pressures were observed immediately before reaching the final water saturation. It is realised that this indicates some degree of mixed wettability, whereas Land assumes cores to be water wet. Whether this may affect the procedure for adjusting the connate water saturation, has not been investigated.

The high negative capillary pressures were ignored in the simulator. Sensitivities demonstrated the impact of this simplification to be minimal.

\section{Displacement Performance}

\subsection{Fine Grid Model Results}

To investigate the displacement process a fine grid cross-sectional simulation model was constructed. The model represents a section from crest of the field towards the northorn end of the field. It also formed the linsis for generation of inter grid block pseudo rolative 
permeabilities used in the full field model, as explained below. Simplified rock properties were used in the fine grid model. The new relative permeabilities based on the steady state and centrifuge data were used. Water injection and oil production rates were selected to yield a displacement front velocity as expected in the field. Saturation distributions after 4.8 and 7.3 years of production are shown in Figs. 8 and 9. The "tongue" of oil lagging behind the displacement front underneath the cap rock should be noted. This feature is caused by the tail of very low oil relative permeabilities before reaching the residual oil saturation. Oil with very low mobility is initially bypassed by water. However, in the highly permeable sands this oil still has sufficient mobility to more slowly vertically upwards, driven by gravity forces. It then collates underneath the cap rock to form the observed oil tongue. In this location the oil relative permeability increases due to the higher oil saturation, allowing flow in the updip direction towards the producers. On this basis it appears that gravity drainage will be an active drive mechanism in the Draugen reservoir. In fact the displacement can be split into two distinctive mechanisms: a normal laterally moving frontal displacement followed by the vertical gravity drainage in the region behind the displacement front.

\subsection{Inter Grid Block Pseudos}

The Draugen full field simulation model has a regular areal grid with a grid block size of $400 \mathrm{x}$ $400 \mathrm{~m}$ within the oil bearing area. The main Rogn 1 unit is subdivided into 7.3 metre thick simulation layers. Based on the fine grid simulations it is obvious that complete fluid dispersion cannot be assumed within an area equivalent to the grid block size of the full field model. The development of pseudo relative permeabilities was therefore required. These pseudos would have to be constructed in such a way that they would describe both the lateral displacement and the gravity drainage behind the front.

To develop the pseudos a simple analytical program was applied. The program assumes segregated flow and allows a formation dip as well as a dipping fluid contact inside the grid block. A similar procedure for a gravity-stable gas/oil system was reported by $\mathrm{Ypma}^{3}$. In the Draugen fine grid model the fluid contact dip was found to be about 10 degrees, an order of magnitude higher than the formation dip. The relative permeabilities in each flow direction are proportional to the grid block interface areas covered by each of the phases and the end point relative permeability of each phase. This principle is used for all six flow directions out of a grid block, not only the principal displacement direction represented in the fine grid model simulations. Emphasis is on imbibition, but the program also calculates pseudo relative permeabilities for the drainage cycle. Pseudo capillary pressures are calculated based on the rock values and the dimensions of the grid block, assuming vertical equilibrium.

The program used for developing the pseudos does not include gravity drainage, which is a separate process. Some degree of trial and error was involved in finding the best way of representing this process in the pseudo function. The final solution was to assume an initial displacement process with a residual saturation of $40 \%$. This is followed by the gravity drainage process, represented by a tail manually added to the oil pseudo relative permeability. The resulting function in the principal flow direction is shown in Fig. 10. A coarse grid model with grid blocks of the same size as in the full field model was used for validation of the derived pseudo relative permeabilities. The main parameter for comparison was saturation distribution, but also pressure distribution was checked. The average water saturation of grid block columns along the length of the coarse and fine grid models at 7.3 years is shown in Fig. 11. Good agreement is demonstrated between the two models, thus proving the validity of the pseudos.

\section{Well Performance}

\subsection{Benefits of Horizontal Wells}

In the Plan for Development and Operations the field was assumed to be developed with 6 . deviated platform wells, 4 of which would be drilled during the initial development and the two last ones would maintain well capacity after water break through. In addition two appraisal wells (wells 3 and 5 in Fig. 3) would be hooked up as subsea producers to provide early production. One of these wells would also drain a separate sub-structure, Rogn South, at the southern extremity of the field. All production wells were planned to be completed with internal gravel pack completions.

In 1992 a study was carried out to evaluate the potential benefits of completing the producers as horizontal wells. Reservoir performance, well inflow and tubing lift were considered. Based on the inflow and lift considerations it was concluded that a significant increase in well productivity could be obtained. The planned 
well capacity could be increased from 5000 to $7500 \mathrm{Sm}^{3} / \mathrm{d}$

The Draugen plattform producers are located at the crest of the field where the bottom Lower Spekk shale or the low permeable Rogn II formation rises above the oil/water contact. This implies that water coning was not expected to be a problem at initial conditions. However, upon arrival of the water displacement front water will cone into the producers. To evaluate this process for vertical and horizontal wells, fine grid element models were applied. Grid sensitivities were carried out to ensure that the grid was sufficiently fine to yield reliable results. In addition to simulation analytical formulae were employed to assess the critical rate to coning. These formulae indicated that the expected production rate would by far exceed the critical rate, both for vertical and horizontal wells. However, in the simulator significant differences in coning performance were seen between the vertical and horizontal wells. Upon arrival of the water front the cone would form faster in the vertical well than in the horizontal well, leading to earlier water break through and higher water cut in a vertical well. This is explained by the large volume of the cone underneath the entire length of the horizontal completion requiring longer time to form than the narrow cone below the vertical completion.

The saturation distributions around the well is seen in Fig. 12. As can be seen the cone is most severe close to the "heel" of the horizontal section, resulting from the fact that the largest contribution to production originates from this part of the well. The internal friction along the horizontal completion is the reason for this feature.

\subsection{Well Pseudos}

In order to properly model the near-well effects in the coarse grid full field model, pseudo relative permeabilities had to be generated for the flow from the well grid block into the well bore. This way it is possible to represent the cone which actually occurs inside the volume of the well block. A computer program was used to translate the fine grid model results into the appropriate pseudo function. The program extracts the pore volume weighted oil phase pressure, $p_{0}$, and the water saturation, $S_{w}$, within a region of the fine grid model corresponding to the well grid block of the full field model. The bottom hole pressure, $p_{w f}$, and the oil and water rates, $q_{0}$ and $q_{w}$, are also extracted. Soon after production start-up (before water encroachment) the well pseudo relative permeability is assumed to be equal to the rock relative permeability at initial connate : water saturation, $\mathrm{k}_{\mathrm{ro}}\left(\mathrm{S}_{\mathrm{wc}}\right)$. At this point a constant. is calculated:

$$
C=\left(p_{o}-p_{w f}\right) \cdot k_{r o}\left(S_{w c}\right) / q_{0}
$$

At subsequent times the oil pseudo relative permeability was calculated as follows

$k_{\mathrm{ro}}=\frac{\mathrm{q}_{\mathrm{o}} \cdot \mathrm{C}}{\mathrm{p}_{\mathrm{o}}-\mathrm{p}_{\mathrm{wf}}}$

The water relative permeability was calculated in a similar fashion, taking into account the differences in viscosity and formation volume factor:

$k_{r w}=\frac{q_{w} \cdot \mu_{w} \cdot B_{w} \cdot C}{\mu_{w} \cdot B_{o}\left(p_{o}-p_{w f}\right)}$

The pseudo relative permeabilities as calculated in this manner for a horizontal well are shown in Fig. 13. However, when using these pseudos in the completed grid blocks of a coarse grid model it was not possible to reproduce the fine grid model results. The buildup of the water cone in the coarse grid blocks underneath the well was too slow. A separate pseudo function favourable to vertical water flow was introduced in these grid blocks (See Fig. 14). This was combined with a lower vertical permeability in these blocks. In this manner the good match illustrated in Fig. 15 was achieved.

\subsection{Early Production Wells}

The Draugen development plan included the use of two appraisal wells to the south of the platform as producers. The southernmost of these (denoted 55 in Fig. 2) is located on the Rogn South substructure which has a maximum oil column of some 16 metres. The original plan was to use the test completion also for production. However, based on simulation studies early water breakthrough and high water cut was expected in this well. A new study was therefore carried out comparing the performance of the original completion with that of a horizontal sidetrack of the well. It was demonstrated that the horizontal well would perform significantly better than the original completion. Based on the incremental production for the time until the platform capacity could be filled from platform wells, it was justified to sidetrack the well as a horizontal well.

The other appraisal well, the so-called Southern Oil Producer (denoted 53 in Fig. 2), was not 
converted to a horizontal well since early water production was not expected in this well and flowline restriction prevented utilisation of additional capacity.

Pseudo relative permeabilities for the Rogn South well and the Southern Oil Producer were constructed in the same manner as for the platform wells. This ensured realistic well performances in the full field model.

\section{Field Performance}

\subsection{Prediction}

The Draugen full field model which incorporates the features discussed above, has been used in the evaluation of development scenarios.

The model has a regular $400 \mathrm{~m} \times 400 \mathrm{~m}$ grid covering the area indicated in Fig. 2 and 7.3 metre thick layers in the main part of the reservoir.

The production history to date and the predicted future field performance is depicted in Fig. 16. The field rate is constrained at a yearly average plateau level of $15,900 \mathrm{Sm}^{3} / \mathrm{d}$ (increased from $14,300 \mathrm{Sm}^{3} / \mathrm{d}$ in the PDO). The main field recovery (excluding Garn West) amounts to 94 million $\mathrm{Sm}^{3}$. This compares to 67 million $\mathrm{Sm}^{3}$ in the PDO. The increase is attributed to the reduced residual oil saturation and to the reduced and delayed buildup of water production. Hence the new relative permeability model with corresponding pseudos and the introduction of the horizontal wells are the reasons for the significant addition to the reserves.

\subsection{Actual Production Performance}

Production from the two subsea completed appraisal wells started in October 1993. Due to more work having to be done offshore than originally planned the start of platform well drilling had to be delayed about six months. The first platform well came on stream in June 1994. Due to this delay the importance of the early production from the subsea wells was increased.

The Rogn South well has demonstrated a slower water cut development than predicted, thus providing significant additional volumes of early production. The better than expected performance is believed to be related to a tight streak observed immediately below the OWC in the original appraisal well. Due to the small thickness of some 4 metres this was assumed not to be extensive and therefore omitted in the simulation model. A history matching exercise is planned to explain the Rogn South performance and form the basis for predictions.

The platform wells have performed as planned or better. The third well, A-2 (see Fig. 2), tested at a rate of $8000 \mathrm{Sm}^{3} / \mathrm{d}$. In fact the platform wells have proved so prolific that it was decided to drill only three of the four planned wells initially. The three platform wells and the two subsea wells still provide ample well capacity to achieve the planned yearly average field rate of $15,900 \mathrm{Sm}^{3} / \mathrm{d}$.

\section{Plans For The Future}

\subsection{Further Development}

Increasing Draugen production above 15,900 $\mathrm{Sm}^{3} / \mathrm{d}$ is being considered. On this basis additional well capacity may be required, leading to one or two additional platform wells. In the longer term additional wells may also be drilled to maintain well capacity when the water cut increases.

A feasibility study has established that the Garn West substructure can be reached with a well drilled from the platform. In the base case this well is assumed to come on stream in 2007. The well is assumed to be drilled as a horizontal well. A dedicated well pseudo relative permeability function has been developed for inclusion of this well in the full field simulation model. Production from the Garn West well adds 5 million $\mathrm{Sm}^{3}$ to the Expectation Reserves bringing the total field figure to 99 million $\mathrm{Sm}^{3}$.

\subsection{Monitoring}

The Draugen field is essentially developed with a central cluster of production wells supported by water injection at the southern and northern ends of the field. This scheme leads to an expected period of some 10 years with dry oil production before arrival of the water front in the platform area. Only a single well, the Southern Oil Producer is located between the injectors and the platform production wells. Monitoring of the displacement front based on well observations is therefore not possible. To compensate for this, other methods have been considered. These are the use of oil finger prints and time lapse seismic as explained in the following.

The fact that the Draugen oil quality varies strongly across the filed, implies that intrinsic tracers are found in oils from different parts of the field. Analyses have established distinct fingerprints of oils from all Draugen wells, allowing these to be roughly mapped across the 
field. A sampling programme has already been implemented with oil samples being taken and analysed monthly from each well. Although some changes in the fingerprints have already been observed it is too early to draw any conclusions. The next generation full field simulator to be constructed later this year, will allow tracking of the fingerprints, thus providing a history matching parameter which may be used prior to water breakthrough.

Time lapse seismic may be another technique for Draugen reservoir monitoring. Shell has developed a technique for comparing changes in seismic response with changes in grid block saturations. This implies that time lapse seismic can provide history match data for the reservoir simulator. A feasibility study will be carried out to establish the applicability of this technique before considering shooting time lapse seismic.

\section{Conclusions}

1. Relative permeability experiments using steady state and centrifuge techniques yield significantly lower residual oil saturations than unsteady state experiments.

2. Use of the steady state/centrifuge data in fine grid simulation models results in a gravity segregation process taking place behind the displacement front.

3. The use of horizontal wells will result in delayed water cut buildup compared to vertical wells, due to the larger volume of the cone underneath a horizontal well.

4. The new relative permeabilites and the introduction of horizontal wells have increased the expected recovery factor for Draugen from $37 \%$ to $52 \%$ (54\% including Garn West).

5. The use of oil fingerprints and possibly time lapse seismic will provide history match data for the simulator and hence aid in flood front monitoring. This will compensate for the lack of observation wells over the long distances between injectors and producers.

\section{Acknowledgements}

The author wishes to thank colleagues in Norske Shell, and in particular Helge Hammer, who carried out a large part of the technical work behind this paper. The Norske Shell and Shell International management and the Draugen partners are also thanked for permission to publish this paper. The views presented are those of Norske Shell and may not be fully shared by the Draugen partners.

\section{Nomenclature}

$B_{0}$ Oil formation volume factor, res.vol./surf.vol.

$B_{w}$ Water formation volume factor, res.vol./surf.vol.

C Constant - as defined in text

GOR Gas/Oil ratio, $\mathrm{Sm}^{3} / \mathrm{Sm}^{3}$

$\mathbf{k}_{\mathrm{v}} \quad$ Vertical permeability, $\mathrm{mD}$

$\mathbf{k}_{\mathrm{h}}$ Horizontal permeability, $\mathrm{mD}$

$\mathbf{k}_{\text {ro }}$ Oil relative permeability, fraction

$\mathbf{k}_{\mathrm{rw}}$ Water relative permeability, fraction

$\mathrm{p}_{\mathrm{c}}$ Capillary pressure, bar

$p_{0} \quad$ Oil phase pressure, bar

$p_{w f}$ Well bottom hole flowing pressure, $b a r$

$\mathrm{q}_{0} \quad$ Oil flow rate, $\mathrm{Sm}^{3} / \mathrm{d}$

$q_{w} \quad$ Water flow rate, $\mathrm{Sm}^{3} / \mathrm{d}$

$\mathrm{S}_{\mathrm{o}} \quad$ Oil saturation, fraction

$\mathrm{S}_{\mathrm{w}} \quad$ Water saturation, fraction

$S_{\text {or }}$ Residual oil saturation, fraction

$\mathrm{S}_{\mathrm{wc}}$ Connate water saturation, fraction:

$\mu_{\mathrm{w}} \quad$ Oil viscosity, cp

$\mu_{\mathrm{w}} \quad$ Water viscosity, $\mathrm{cp}$

\section{References}

1. Land, C.S: Calculation of ImbibitionRelative Permeabilities for Two and ThreePhase Flow from Rock Properties, SPEJ, June 1968.

2. Honarpour, M: Relative Permeability of Petroleum Reservoirs, CRC Press, Second print 1987.

3. Ypma, J.G.J.: Analytical and Numerical Modeling of Immiscible Gravity-Stable Gas Injection Into Stratified Reservoirs, SPE 12158, SPEJ August 1985. 


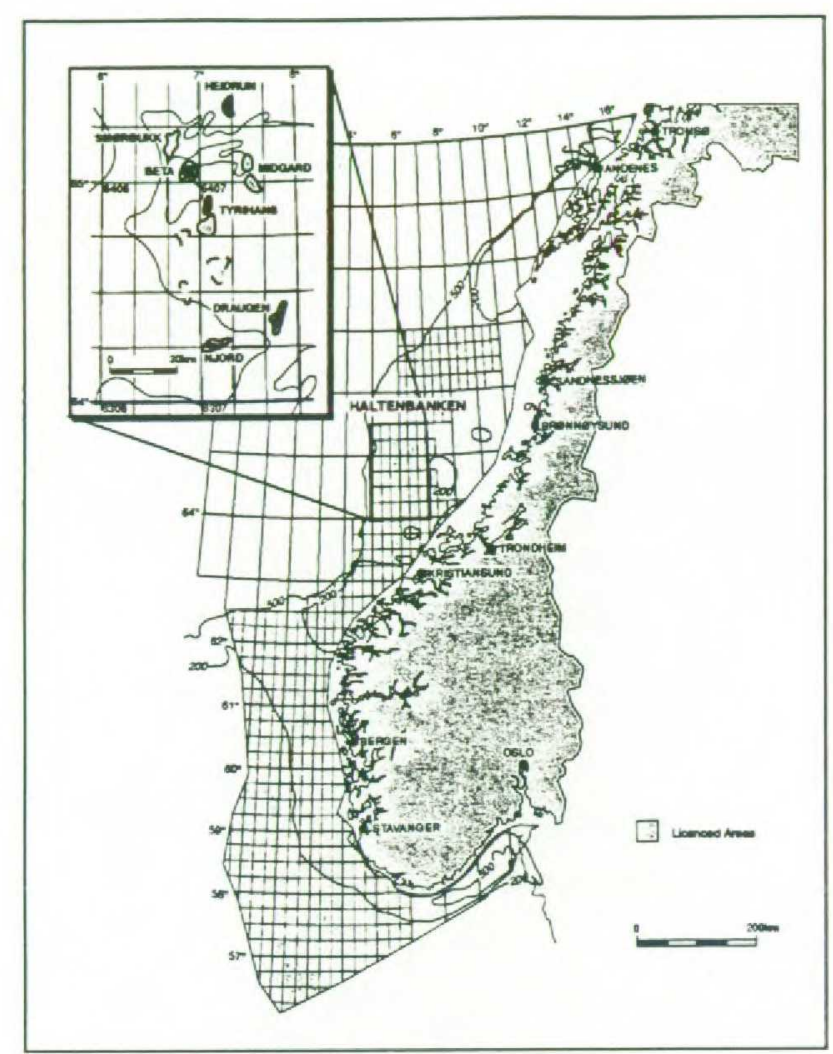

Fig. 1. Draugen location map

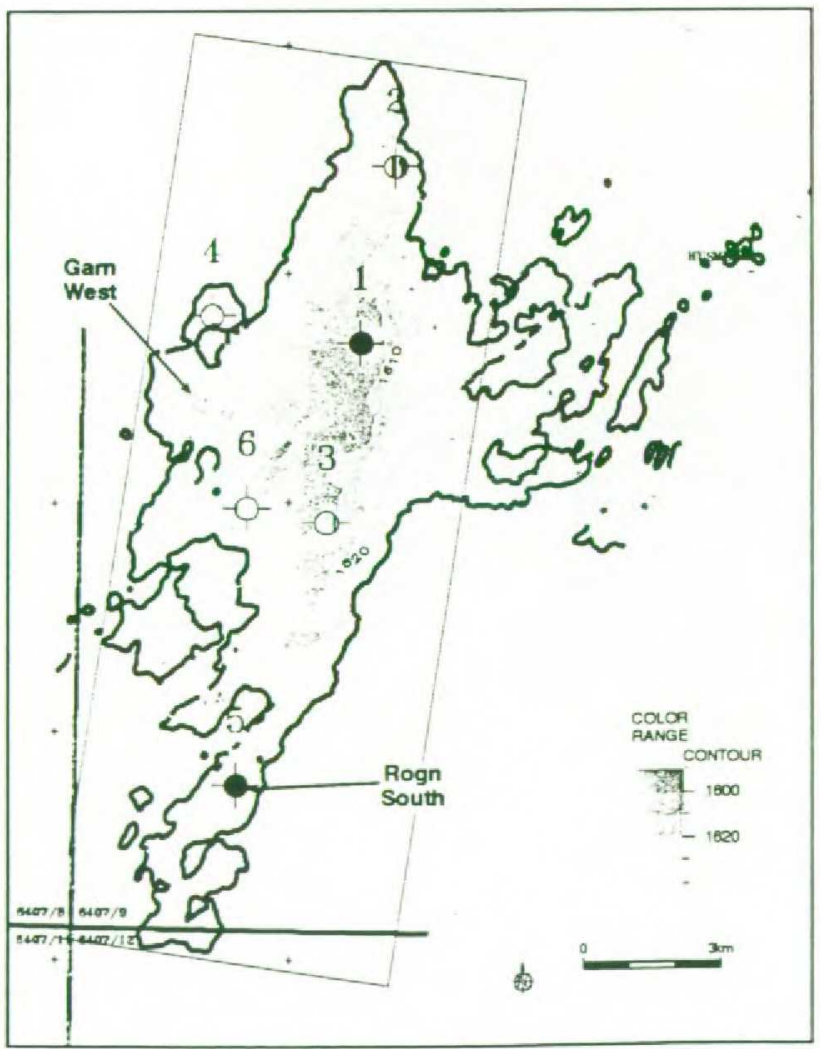

Fig. 3. Draugen top reservoir depth map

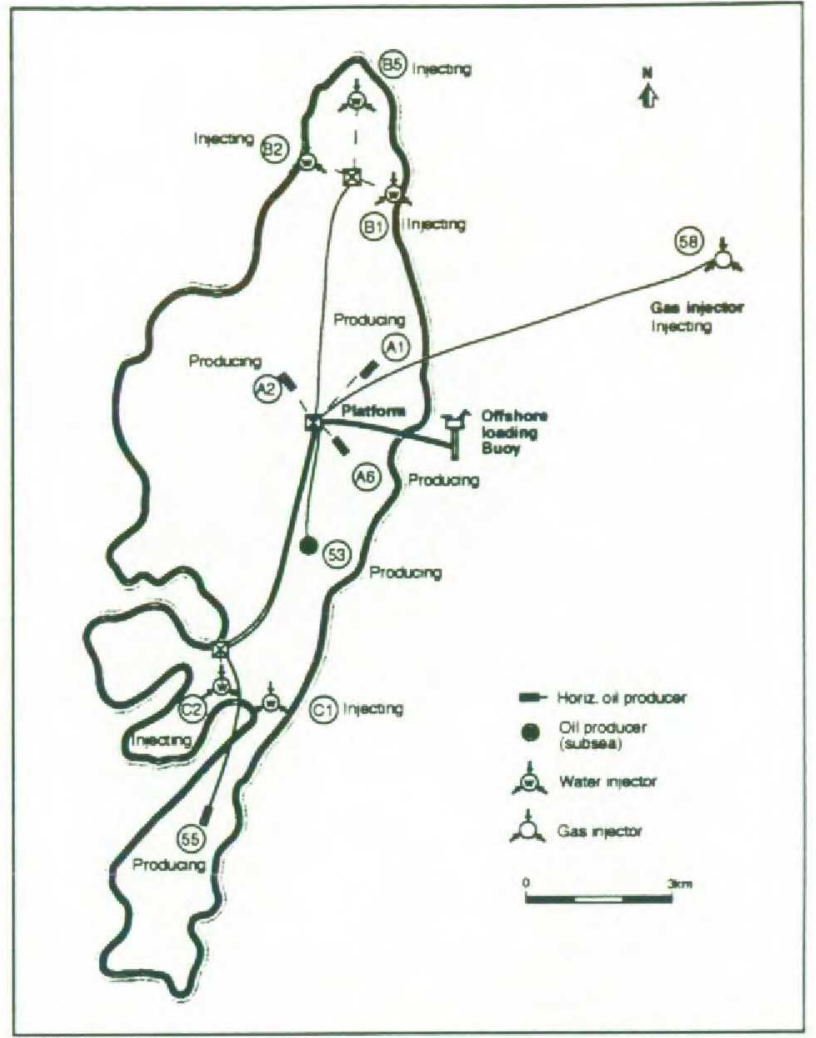

Fig. 2. Status end 1st phase Draugen development campaign December 1994

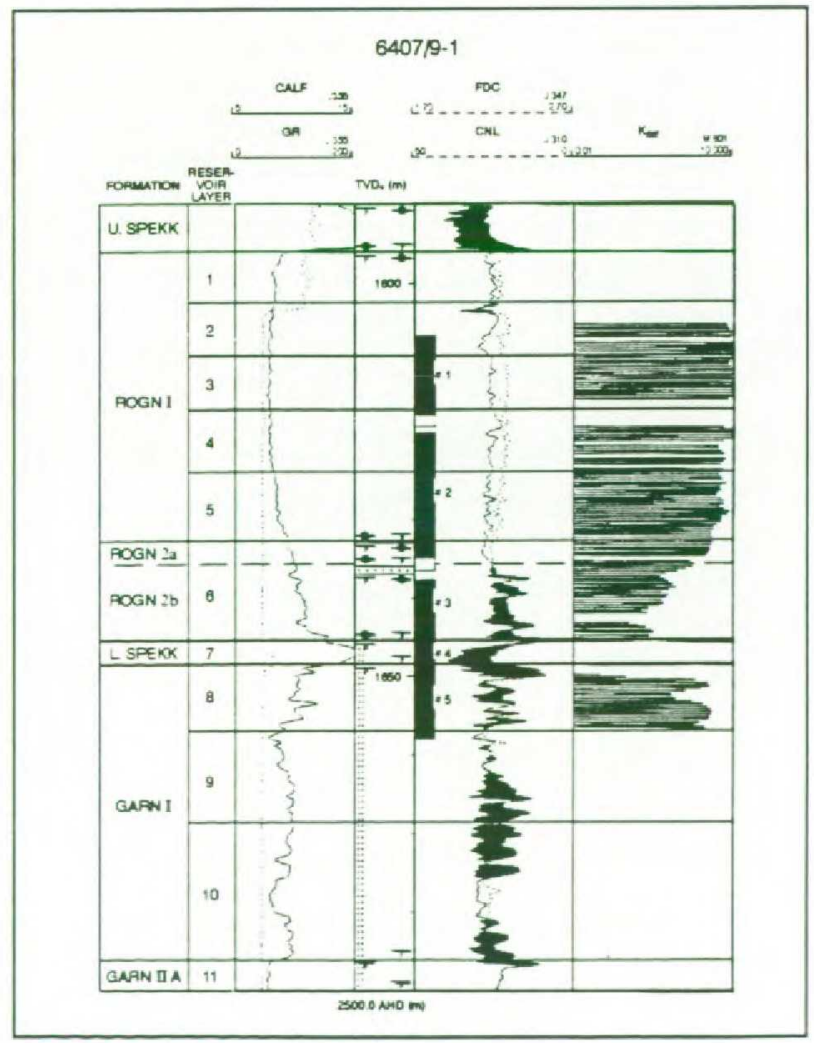

Fig. 4. Draugen type log 


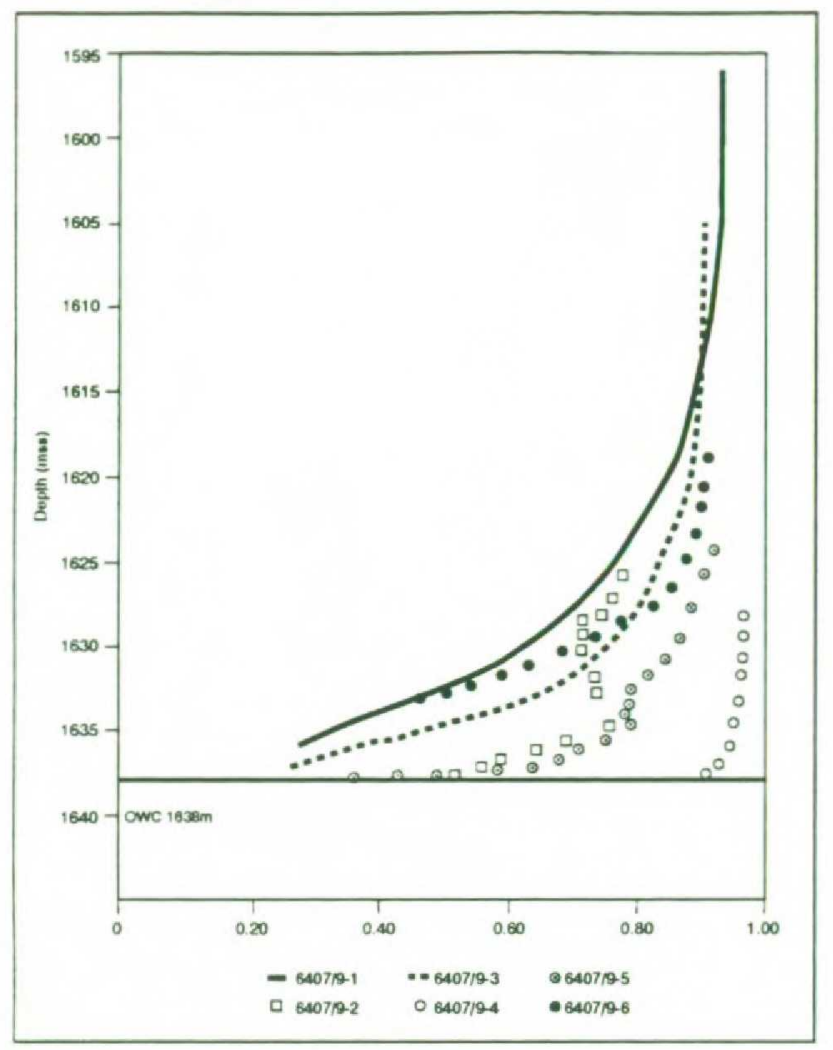

Fig. 5. Oil saturation vs. depth

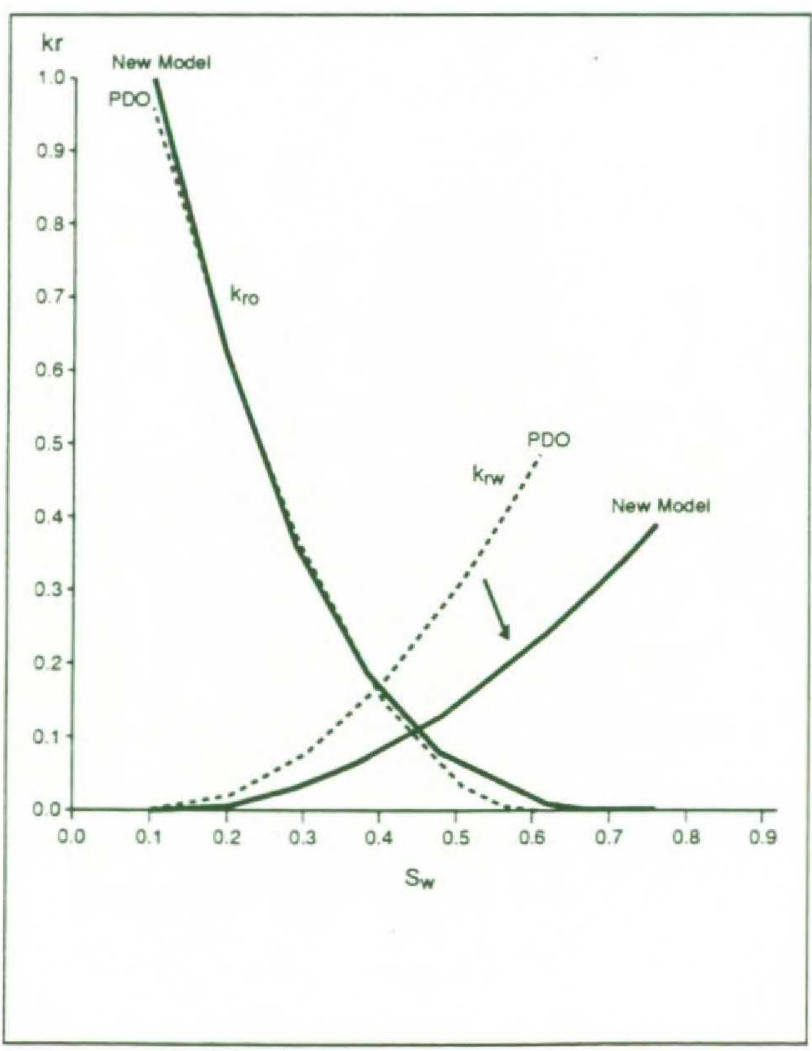

Fig. 7. Relative permeabilities Rogn I

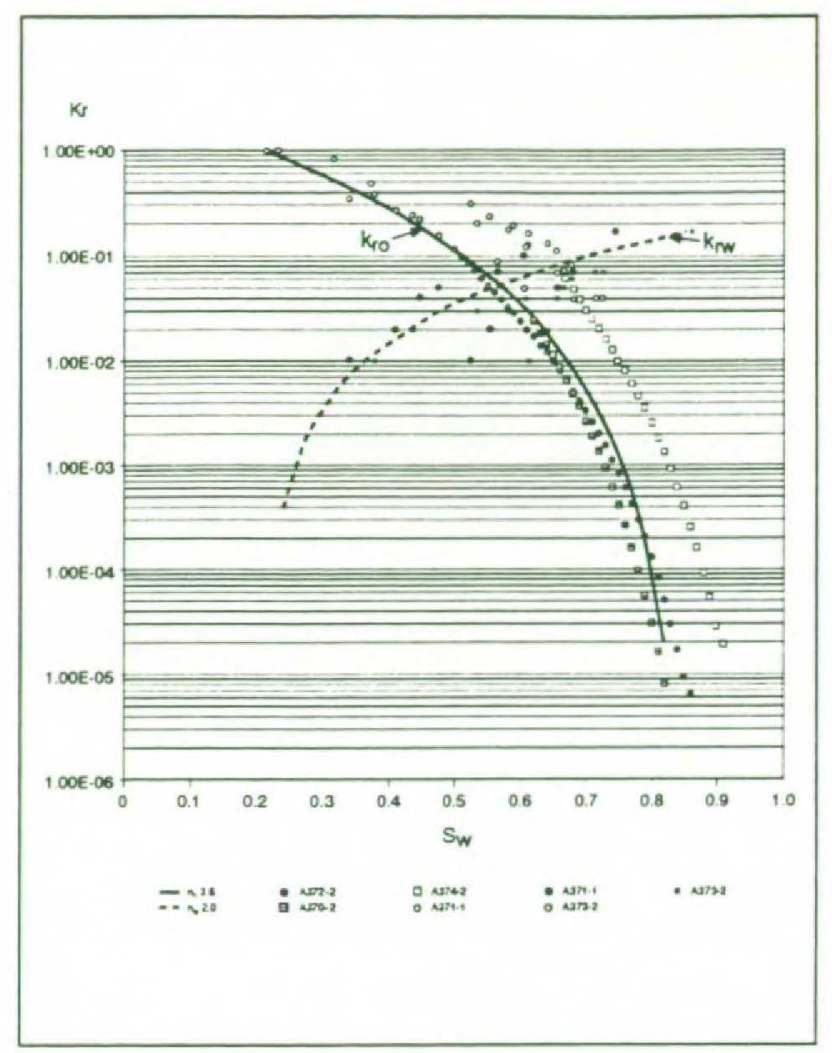

Fig. 6. Steady state and centrifuge measurements

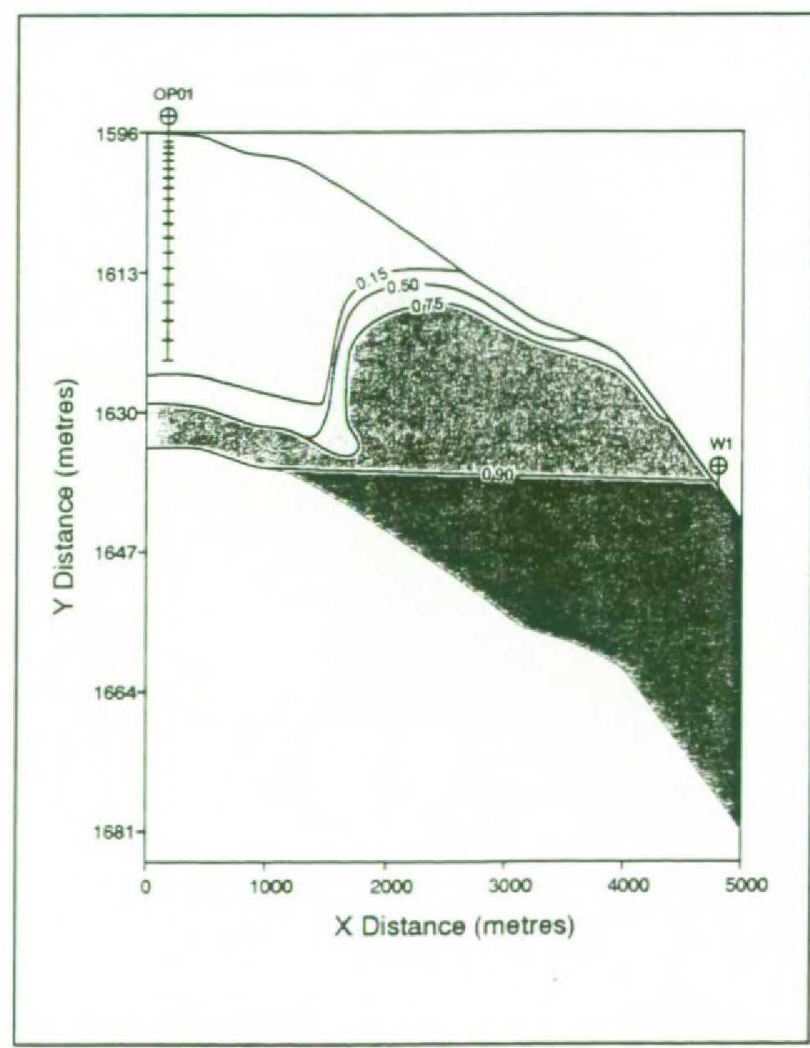

Fig. 8. Fine grid reference model - 4.8 years Water saturation 


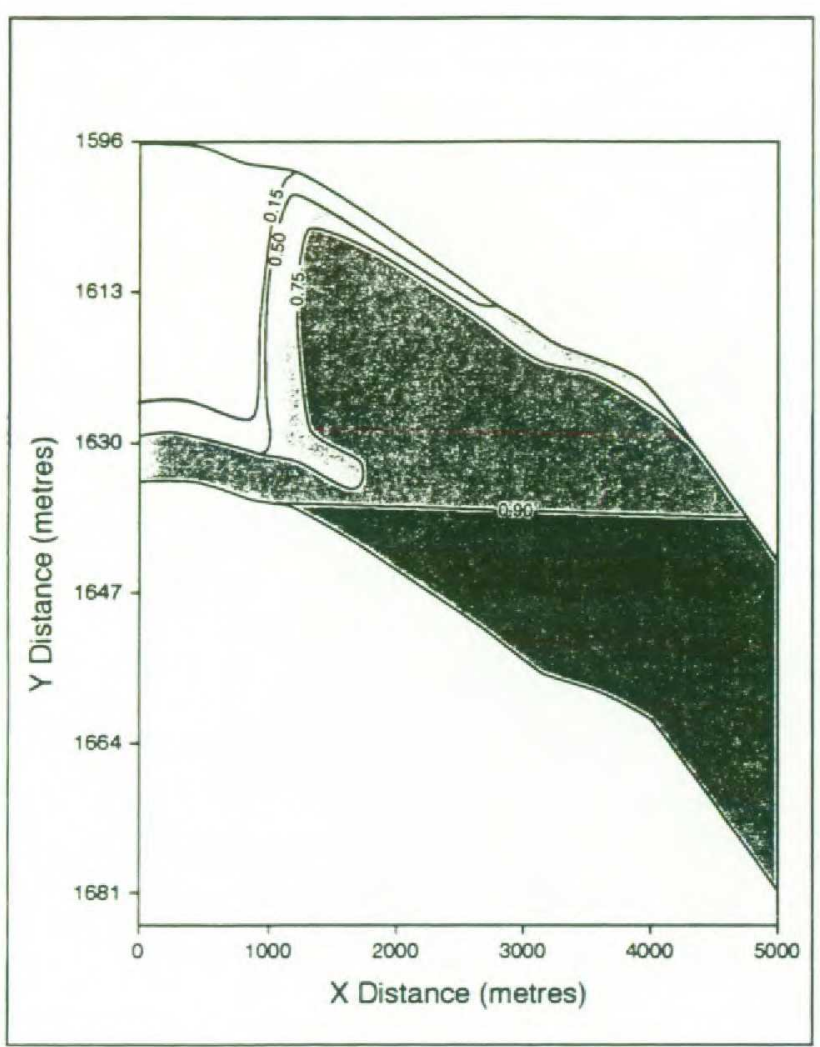

Fig. 9. Fine grid reference model - 7.3 years Water saturation

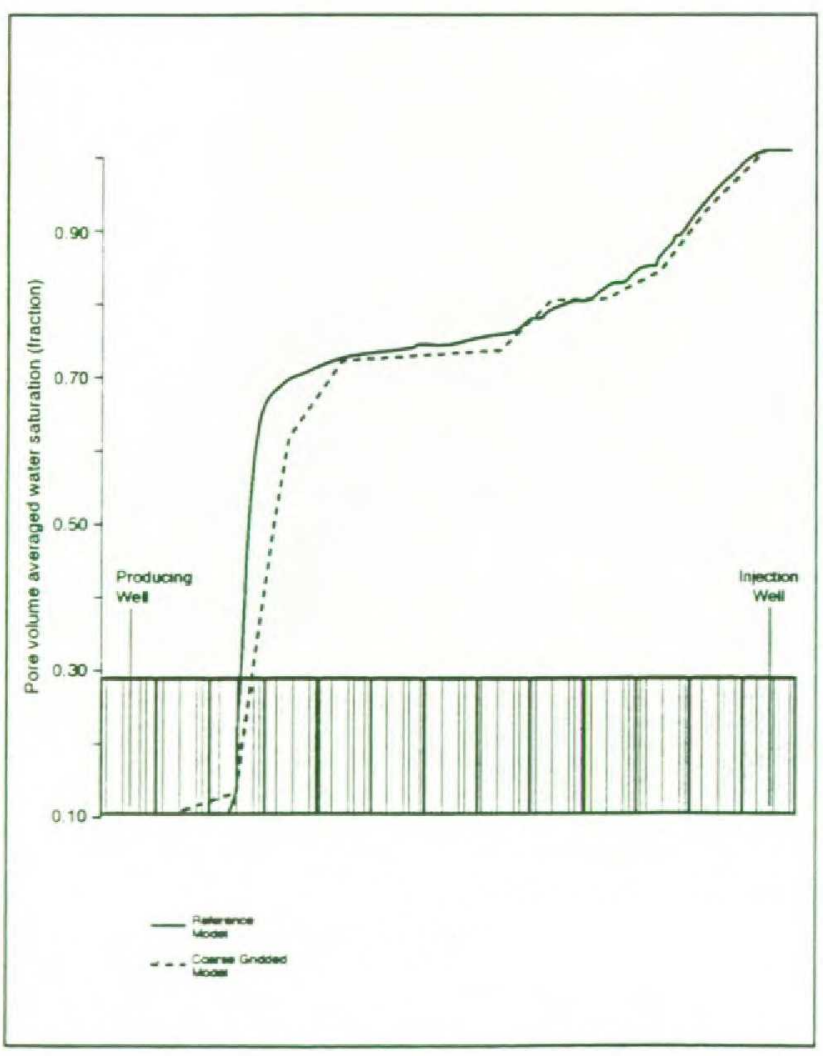

Fig. 11. Match between the coarse grid model and the reference model after 7.3 years

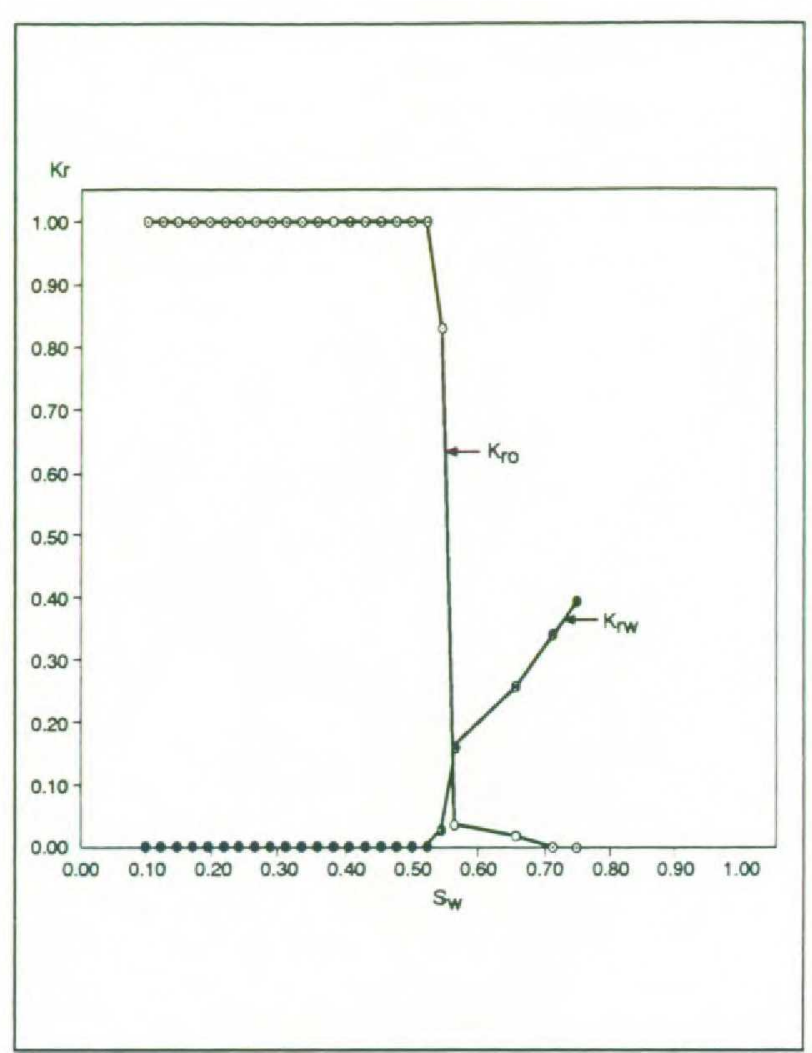

Fig. 10. Oil / water pseudo function - principal flow direction

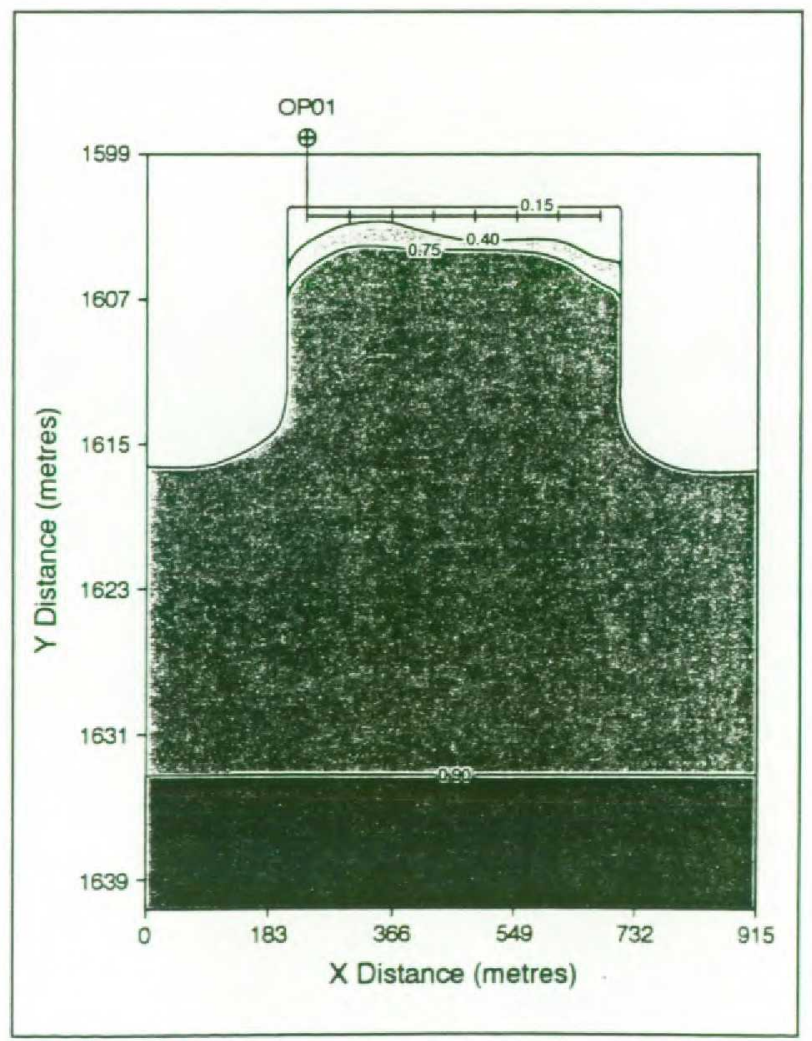

Fig. 12. Very fine grid horizontal well model water cone along horizontal section 


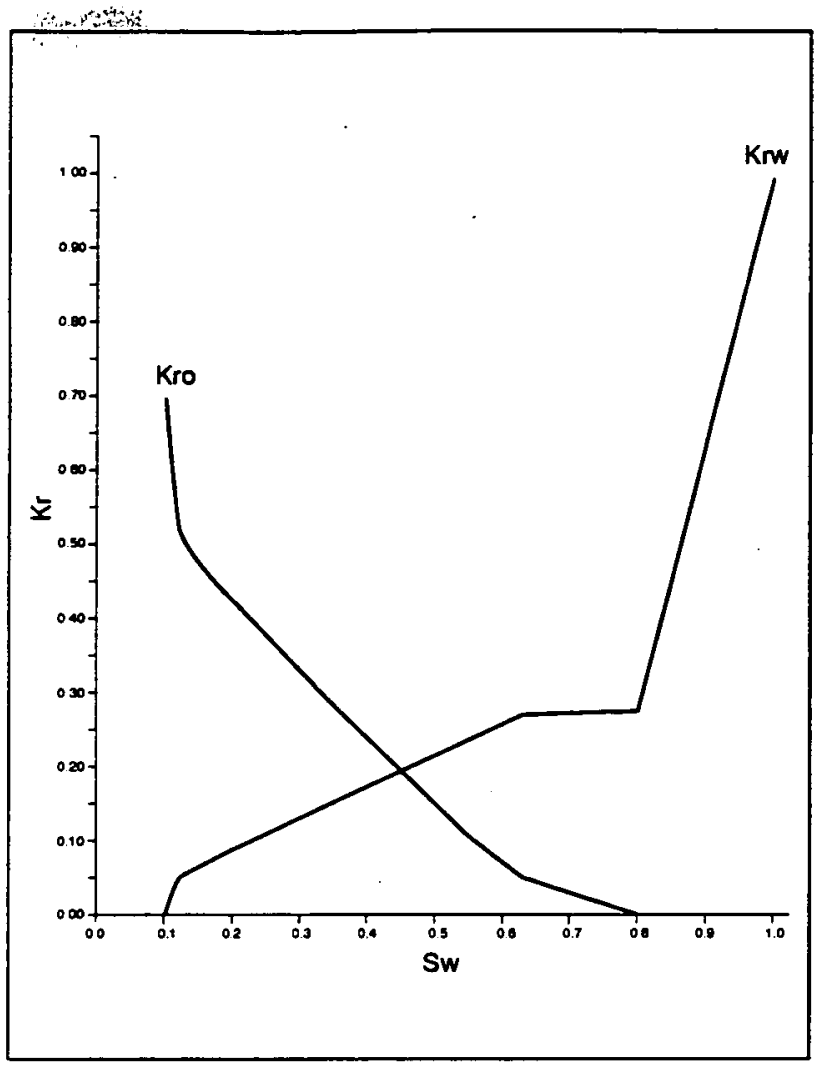

Fig. 13. Pseudo relative permeability horizontal well

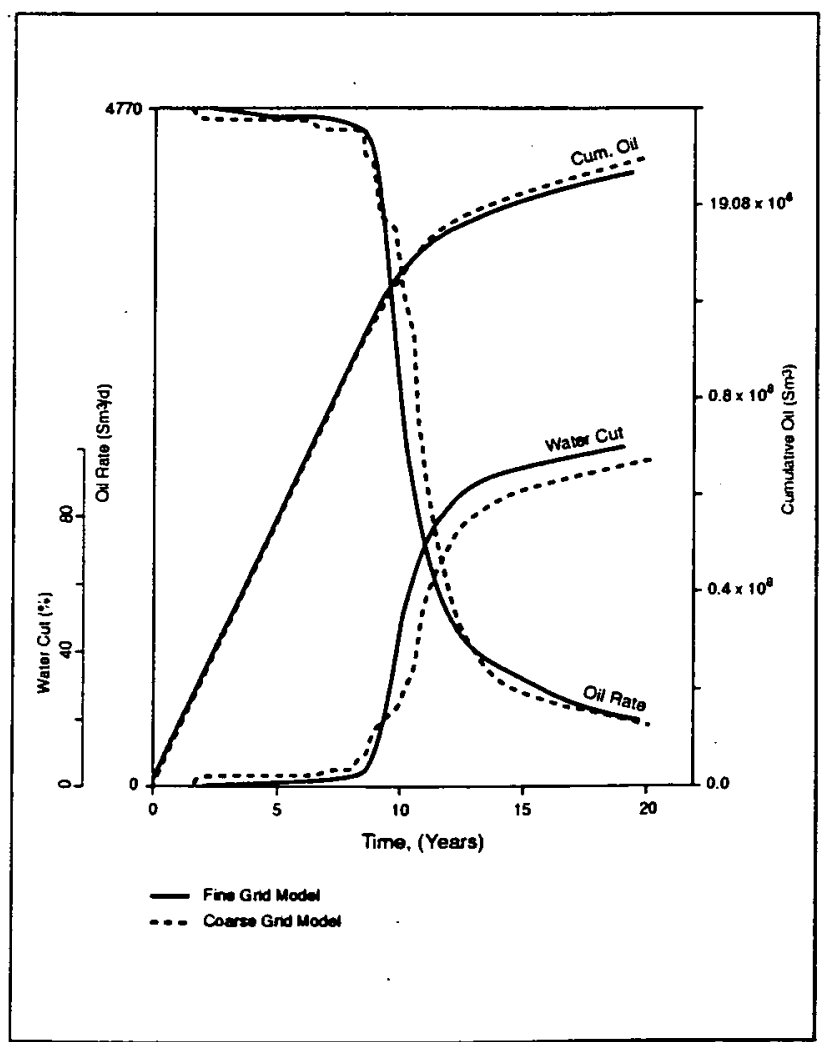

Fig. 15. Match between fine and coarse grid models, horizontal well

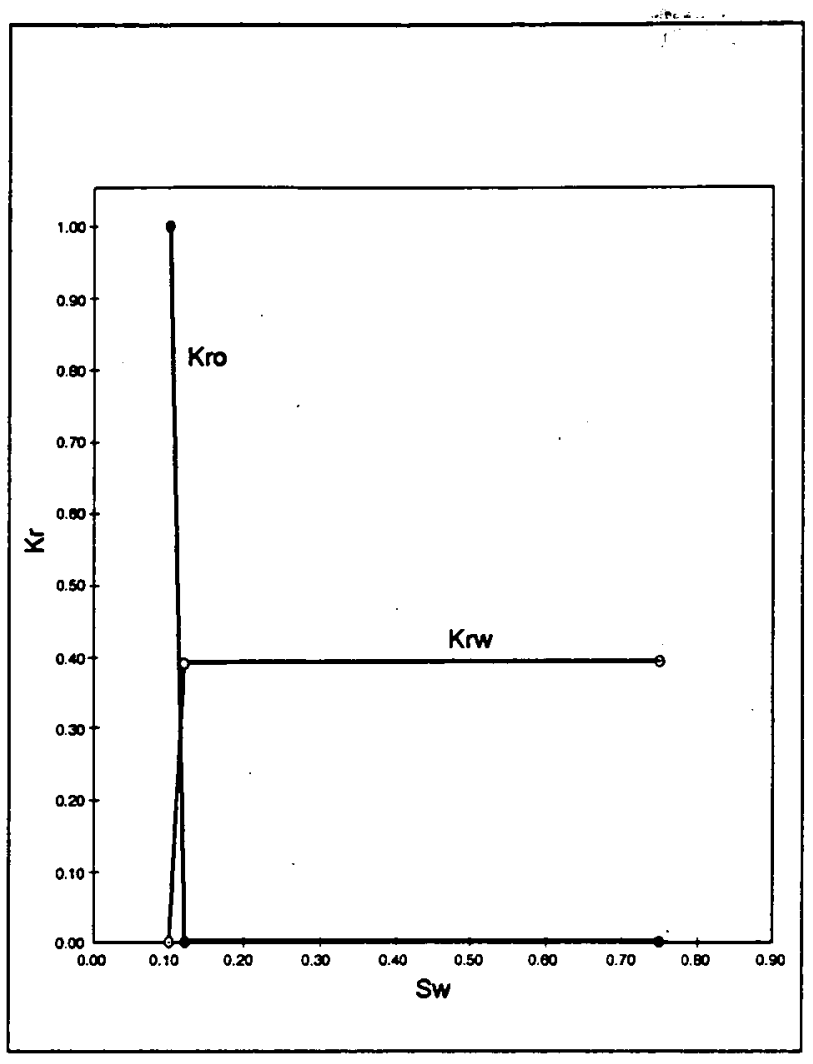

Fig. 14. Well pseudo functions to allow upwards flow of water

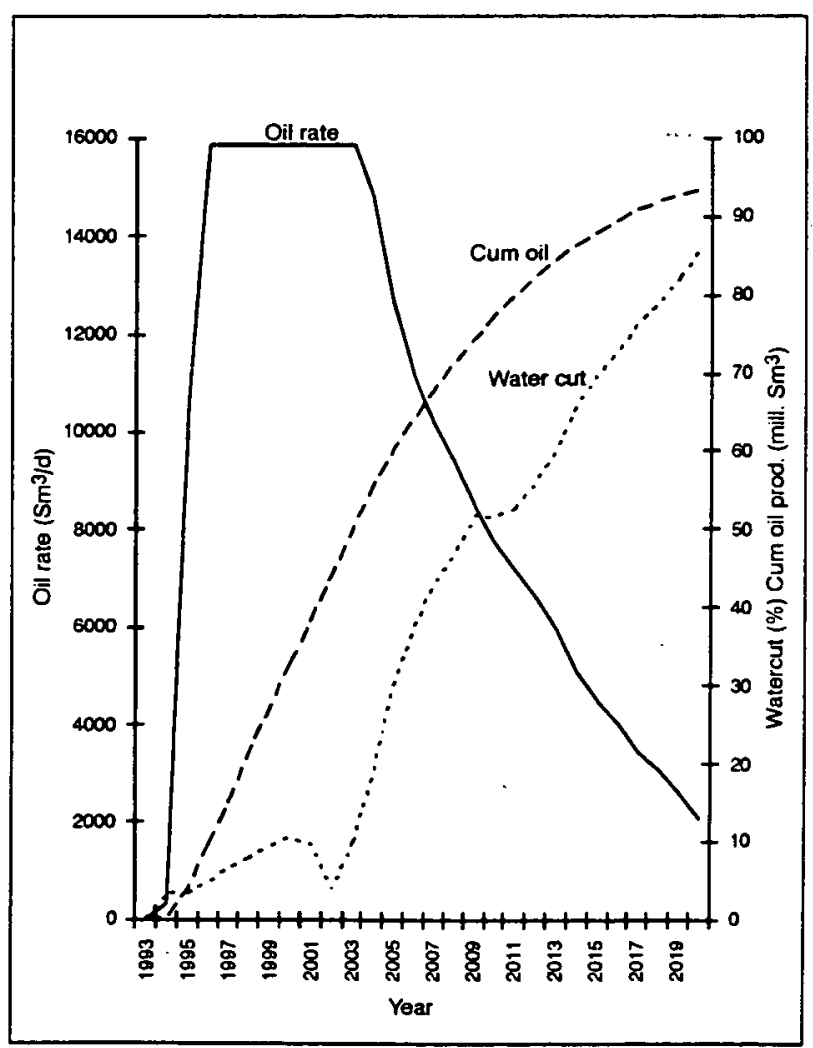

Fig. 16. Draugen main field production profile 